\title{
Birth weight centiles and small for gestational age (SGA) by sex and ethnicity for England and Wales.
}

Anna Freni-Sterrantino UK Small Area Health Statistics Unit, MRC-PHE Centre for Environment and Health, School of Public Health, Imperial College London, London

Priscilla Afoakwah, AngloGold Ashanti Health Foundation, Obuasi, Ashanti, Ghana

Rachel B. Smith MRC-PHE Centre for Environment and Health, School of Public Health, Imperial College London, London, London, UK

Rebecca E. Ghosh UK Small Area Health Statistics Unit, MRC-PHE Centre for Environment and Health, School of Public Health, Imperial College London, London, UK

Anna L. Hansell, Centre for Environmental Health and Sustainability, University of Leicester, Leicester, UK; Small Area Health Statistics Unit, MRC-PHE Centre for Environment and Health, School of Public Health, Imperial College London, London, UK; Imperial College Healthcare NHS Trust, London, UK

Corresponding author

Anna Freni Sterrantino

UK Small Area Health Statistics Unit (SAHSU), Department of Epidemiology and Biostatistics School of Public Health, Imperial College London, St. Mary's Campus, Norfolk Place, London W2 1PG, U.K Telephone 02075943276

Email: a.freni-sterrantino@imperial.ac.uk 


\section{Acknowledgments}

The authors are grateful to Professor Tim Cole (University College London) for useful comments and suggestions and to Margaret Douglass for data extraction.

\section{Contributors}

AFS drafted the paper and supervised the statistical analysis conducted by PA. All the authors provided intellectual input, interpreted the results, and helped to revise the manuscript. All authors approved the final version of the manuscript and agreed to be accountable for all the aspects of the work in ensuring that questions related to the accuracy or integrity of any part of the work are appropriately investigated and resolved. AFS is the guarantor of this paper.

Competing Interests None declared.

\section{Ethics and Information Governance statement}

This study uses SAHSU data, covered by national research ethics approval from the London-South East National Research Ethics Committee - reference 17/LO/0846. Data access is covered by the Health Research Authority - Confidentiality Advisory Group under section 251 of the National Health Service Act 2006 and the Health Service (Control of Patient Information) Regulations 2002 HRA CAG reference: 14/CAG/1039. Identifiable information has only been used under strict data sharing agreements with the data providers. SAHSU does not have permission to supply data to third parties. 


\section{Funding}

The UK Small Area Health Statistics Unit (SAHSU) is part of the MRC-PHE Centre for Environment and Health, which is supported by the Medical Research Council (MR/L01341X/1) and Public Health England (PHE).

The research was funded/part funded by the National Institute for Health Research Health Protection Research Unit (NIHR HPRU) in Health Impact of Environmental Hazards at King's College London in partnership with Public Health England (PHE) and Imperial College London. The views expressed are those of the author(s) and not necessarily those of the NHS, the NIHR, the Department of Health \& Social Care or Public Health England. 


\section{ABSTRACT}

Objectives To construct UK Ethnic Birth Weight Centiles (UK-EBWC) for gestational age and cutoffs for small for gestational age (SGA) for England and Wales and to evaluate the SGA misclassification using the UK centiles.

Design Analysis of national birth data.

Participants All live singleton births in England and Wales in 2006 to 2012, as recorded by the Office for National Statistics (ONS) and birth registrations, linked with National Health Service (NHS) into Numbers for Babies (NN4B).

Main Outcome Measures Both sex-specific and ethnicity-sex-specific birth weight centiles for gestational age, and ethnicity-sex-specific SGA cut-offs. Centiles were computed using the Generalized Additive Model for Location, Scale and Shape (GAMLSS).

Results Our sex-specific centiles performed well and showed an agreement between the expected and observed number of births below the centiles. The ethnicity-sex-specific centiles for Black and Asian presented lower values compared to the White centiles. Comparisons of sex-specific and ethnicity-sex-specific centiles shows that use of sex-specific centiles increases the SGA diagnosed cases by 50\% for Asian, 30\% for South Asian (Indian, Pakistani and Bangladeshi) and 20\% for Black ethnicity.

Conclusions The centiles show important differences between ethnic groups, in particular the $10^{\text {th }}$ centile used to define SGA. To account for these differences and to minimize misclassification of SGA, we recommend the use of customized birth weight centiles . 
Keywords: Birth weight, Ethnicity, Small for gestational age, birth weight centiles, UK charts 


\section{What is already known on this topic?}

- Identifying babies who are small for gestational age (SGA) helps identify growthrestricted newborns who may be at risk of immediate and long-term morbidity.

- Some ethnic minority groups are characterized by lower birth weights as compared to white ethnicity.

- Currently available birth weight centiles for the UK are sex-specific only, and do not reflect potential (constitutional) differences throughout gestation by ethnicity

\section{What this study adds}

- This study provides ethnicity-sex-specific birth weight centiles derived from national birth records in 2006-12 in England and Wales for 4,927,889 births.

- Produces centiles and cut-offs that can be used in epidemiological studies and inform clinical practice.

- Ethnic-specific centiles and thresholds may avoid misclassifying ethnic minority babies (particularly South Asians) as SGA and subsequently reduce unnecessary interventions, organizational (hospital) costs and parental anxiety. 


\section{INTRODUCTION}

Birth weight is an important indicator for fetal growth and neonatal health in both clinical and perinatal research . Low birth weight (<=2500 g) and very low birth weight $(<1500 \mathrm{~g})$ predict possible future morbidity, but these measures do not distinguish between small babies born early and small babies who grew poorly in utero. Small for gestational age (SGA), defined as a birth weight below the $10^{\text {th }}$ centile for gestational age, is a commonly used measure which adjusts birth weight for gestational age and helps improve the identification of poor growth in utero in both clinical practice and epidemiological research.

In 2009, new UK-WHO growth charts for children aged 0-4 years (1) were developed by the Royal College of Paediatrics and Child Health, which replaced other existing population birth weight centile charts in the United Kingdom (2-4). In 2011, revised birth centiles were released using data from five studies from 1983-1993(5), with less than 10,000 births of which over $80 \%$ were from the East of England (around Cambridge).

Updated UK birth weight centiles were published in 2017 by the MBRRACE programme (Mothers and Babies: Reducing Risk through Audits and Confidential Enquiries across the UK (Norris, Seaton et al. 2017). Centile were constructed using 1.3 million birth records for 2013-14 from the National Health Service (NHS) numbers for babies (NN4B), with centiles being higher than UKWHO. However, these updated centiles did not account for ethnicity, whereas evidence has shown that ethnic-specific birth weight charts have improved prediction of neonatal morbidity and mortality $(6,7)$. 
The UK Millennium Cohort Study (8) found that birth weight distributions differ by ethnic group with White new-borns being heavier than Black and Asians. Differences in birth weight for Black and Asians (Pakistani and Bangladeshi) were explained by socio economic factors, but for Indian and Bangladeshi infants differences were associated with maternal and infant factors. That South Asian ethnicity babies have lower birth weight has been observed in other studies conducted in Canada and Netherlands $(9,10)$. There is evidence $(11-13)$ that the observed South Asians lower birth weight is explained by the physiological characteristics, rather than pathological reasons. In this perspective, several researchers (14-18) recommend the use of customized charts to reduce antenatal care by improving the distinction between physiological and pathological variation in new-borns birth weight.

In UK, ethnic birth weight centiles for ethnic minorities living are based on small and outdated data, and may not be representative of the current South Asian/Pakistani population (19). In addition, due to the lack of ethnic-specific centiles researchers rely on birth weight centiles from other countries or calculate centiles within their own study population to specify SGA $(20,21)$.

In this study, we provide the sex-specific and ethnicity-sex-specific birth weight centiles (UKEBWC) for white, Black and Asian and South Asian ethnicity births, including the cut-offs for the $10^{\text {th }}$ centile used to define SGA, based on over 4 million records from England and Wales.

\section{METHODS}

\section{Data}


We included all live singleton births in England and Wales from January $1^{\text {st }}, 2006$ to December 31 $1^{\text {st }}, 2012$ using data from the ONS (Office National Statistics) Birth Registrations and NHS Numbers for Babies project (NN4B). The two datasets were linked to produce an enhanced birth registrations to include gestational age and ethnicity from NN4B, with $99.8 \%$ of NN4B records linked with a registration record using the NHS number (22). The final dataset includes: the year of birth, sex, birth weight, gestational age in completed weeks, and baby's ethnicity (as reported by the mother).

\section{Data cleaning}

The analysis was restricted to singleton live births occurring between 24 and 42 weeks' gestation. In the data cleaning process, we removed multiple births, implausible birth weight gestational age combinations, missed ethnicity (supplementary material Figure 1). We then split the births into four ethnic groups (supplementary material table 1): White, Asian, Black and Other, to investigate variation in birth weight. Additionally, we split Asians into South Asians (Indian, Pakistani and Bangladeshi) and other Asians (any other Asian background) to examine whether South Asians require separate birth weight reference values from all Asians (19). For each subset defined by ethnicity, sex and gestational age, we excluded birth weight outliers using Tukey fences. Tukey fences is a robust method as it makes no distributional assumptions (23), the lower cut-off is defined as the first quartile minus twice the inter-quartile range (2lQR) and the upper cut-off is the third quartile plus 2 IQR. We removed these outliers separately for the sex-specific charts and for the ethnic group specific charts, for the latter we removed outliers after splitting (table 1) by ethnic group (White, Asian and Black). We excluded the 'Other' ethnic group (6.9\% of births) from 
the analysis as it does not represent a meaningful homogeneous group for analysis and excluded births with missing ethnicity information.

\section{Statistical Analysis}

We computed summary statistics and outlined the density plot for each sex and ethnicity-sex subsets. For each of these subsets, to estimate birth weight charts we used the Lambda-MuSigma (LMS) that models mean, standard deviation, skewness. As we observed kurtosis in the data, we also fitted a second model, the Box-Cox Power Exponential (BCPE) using Generalized Additive Model for Location, Scale, and Shape (GAMLSS) (24). The BCPE is similar to LMS, but additionally models kurtosis as a fourth parameter. We compared the two fits: at extremes centiles (1\% and 99\%), where they could possible diverge, and using a measure of the model quality (generalized Akaike Information Criterion (GAIC)). Both methods showed that BCPE

presented a better fit, so we used this for our analyses. Based on GAMLSS output, we computed birth weight centiles used in clinical practice, rounded to $0.4^{\text {th }}, 2^{\text {nd }}, 9^{\text {th }}, 25^{\text {th }}, 50^{\text {th }}, 75^{\text {th }}, 91^{\text {st }}, 98^{\text {th }}$, 99.6 $6^{\text {th }}$. The centiles are all two-thirds of an SD (standard deviation) scores apart, as reported by (25). We also included the $10^{\text {th }}$ centile, mostly used to define small for gestational age.

\section{Goodness of fit and comparison}

To verify that the centiles performed well, we computed the observed and expected proportion of births below a given centile and checked if they were in agreement.

\section{Small for gestational age and misclassification rate}


We conducted a graphical inspection of the ethnic group specific and sex-specific curves to look at distributional form, and then assessed the ethnic-sex-specific SGA misclassification using sexspecific centiles for Asian and Black, South Asian and Other Asian. All analyses were conducted in R and using the GAMLSS package (24).

\section{Patient involvement}

Patients were not involved in the development of the research question or the design and conducting of the study.

\section{RESULTS}

\section{Descriptive Results}

There were 4,081,910 live singleton births in 2006-12, with $94 \%$ of births observed between 37 42 weeks. White ethnicity was the most common ethnicity for $74 \%$ of all births, with smaller proportions of Asian, Black or Other births. White babies were heaviest followed by Black, Other Asian, South Asian. Males were heavier on average than females, between 2.7\% (Other Asians) to $3.6 \%$ (White) (Table 1). In figure 1, the density plots for both females and males show that white and sex-specific (all data) are overlapping, while Asian and Black are shifted downwards, this also persist at different centiles levels and gestation weeks (Figure 2).

\section{Performance of centiles}

We report the performance of our sex-specific centiles by comparing the observed vs expected percentage of births below centiles. For the $2^{\text {nd }}$ centile we classified males at $2.03 \%$ and females 
at $2.05 \%$ versus an expected average of $2.28 \%$, and at the $98^{\text {th }}$ centiles $2.62 \%$ and $2.22 \%$ for males and females respectively (Table 2 and supplementary Table 2).

\section{Small for gestational age and misclassification rate}

We compared our ethnic-sex-specific $10^{\text {th }}$ centiles for white, Asian and Black against our sexspecific centiles (see Figure 3). White male and female centiles were close to the sex-specific centiles, whereas differences were seen for Black and Asian centiles. Black ethnicity centiles were $80 \mathrm{~g}$ and $52 \mathrm{~g}$ lower (on average across all gestational weeks) for male and female births respectively compared to sex-specific centiles (Figures 3, supplementary Table 3). For Asian males were $113 \mathrm{~g}$ and females $101 \mathrm{~g}$ were lower and similarly for South Asian males 122g and for females 106g lower (supplementary figure 2 and 3).

Within Asian, South Asian and Other Asian, the largest difference was between Asian and Other Asian births with Asian males heavier by $56 \mathrm{~g}$ than Other Asian births and females $35 \mathrm{~g}$ lower for Other Asian births than Asians (on average across all gestational weeks). The birth weight of the $10^{\text {th }}$ centile for South Asians was the lowest among Asians, especially after 32 weeks. Using sexspecific centiles to assess SGA rather than the ethnic-specific centiles (supplementary table 4), we found that for Asian and South Asian births the percentage of births classified as SGA increased by $50 \%$, whereas for Other Asian and Black there was an increase of $30 \%$ and $20 \%$, respectively. 


\section{DISCUSSION}

We provide sex- and ethnic-specific birth weight centiles)for England and Wales (UK-EBWC) based on a large national births' dataset. These new centiles provide a tool to help assess fetal growth, ethnic-specific centiles and small for gestational age births.

Our sex-specific centiles using the UK-EBWC data for 2006-12 for all live singleton births were similar matching to those published from the MBRRACE group (data not shown) (25), who also used data from NN4B (but for 2013-14) plus stillbirths alive at onset of labor $(n=1,269,403)$. Both MBRRACE and our study centiles showed higher birth weights than those reported in 2009 UKWHO charts (5), in line with observed increased birth weight trends between 2006-2012 as reported in Ghosh (26). The UK-WHO revised charts computed on 9443 births, mostly from East of England (5), were limited and not representative.

These two most recent published sex-specific birth weight centiles for the UK have limitations if used to assess Black and Asian births. Whereas, we were not able to directly compare the percentage of misclassification that would have occurred using the MBRRACE data, given our sexspecific curves were similar to the MBRRACE curves, it is likely that using them will lead to similarly increased counts of SGA cases.

The level of misclassification seen for South Asian births in our study is comparable to that seen in a study conducted in Canada (10) and a study Siri Lanka comparing but using Bangladeshi and European centiles. Maso et al. (27) also demonstrated that only SGA cases identified with a ethnicity based charts were at a risk of actual adverse outcome. Narchi et al. (28) suggested that 
relying on a general population based charts will fail to identify a portion of SGA cases that need actual postnatal care.

Whether the differences in birth weight distribution and mis-classification in SGA diagnosis are imputable to physiological or pathological reasons has been debated (29-31).

These differences in birth weights in ethnic minorities have been observed to be consistent also between immigrant mothers for South Asian and Black in UK when compared to second generations, suggesting differences may be physiological (32-34).

Nevertheless, other studies have reported ethnic differences in birth weight for gestational age even after adjusting for all plausible maternal characteristics at the population level $(6,7)$. Sexspecific birth weight charts such as the UK-WHO and MBRRACE charts imply that one chart fits all babies irrespective of ethnicity. However if ignored, the observed ethnic differences in birth weight reference values increase the misclassification of babies of ethnic minorities as SGA, and this could increase further as changes occur in the population composition (35).

Both the UK-WHO (5) and MBRRACE (Norris, Seaton et al. 2017) present $9^{\text {th }}$ centiles and not $10^{\text {th }}$. The choice to focus on the $10^{\text {th }}$ centile is because most of the studies, used this value as a threshold for SGA case. While we do not expect major difference between the $9^{\text {th }}$ and $10^{\text {th }}$ centiles, the observed discrepancies observed in figure 3 would persists.

Compared to the updated UK centiles (MBRRACE), the results presented here have the advantage of a larger dataset comprising over four million live singleton births. Also, the NN4B data are collected nationally rather than from a specific region (5) or hospital population as used for the 
UK-WHO charts. For each ethnic group and gestational age we had enough observations to compute robust centiles. Our analysis highlights that each ethnic specific curve has its own functional form that supports the need for ethnic-specific centiles.

One of the limitations is that our birth weight reference values were computed without any information on maternal medical conditions during pregnancy. Typically, complicated births are excluded in other studies when constructing reference birth weight charts. It is unknown to what extent this may have influenced our ethnic-specific birth weight reference values. Because of this, the Royal College of Obstetrics and Gynaecologists recommend birth weight charts customized for maternal measures that influence birth weight for clinical use to improve the prediction of adverse neonatal outcomes. However, most population birth weight charts used as a reference for defining SGA births, in epidemiological studies do not hold maternal measures, because these were usually not available on population birth registries (36).

The second limitation is our choice of ethnic groups. We chose three main ethnic groups as this grouping is often used in epidemiological studies $(20,21)$, but these three groups comprise subgroups with many cultural and genetic differences. SGA is often used in environmental epidemiological studies as a binary outcome to detect possible exposure effects on fetal growth. Using centiles including all ethnicities does not fit all births and may lead into an incorrect number of SGA cases especially for specific ethnic groups. Finer ethnic grouping may have shown additional difference between groups as it has been shown that birth weights may differ even within the same ethnic groups $(37,38)$, but more categories would have led to smaller sample sizes and less stable estimates, in particular at lower gestational ages. 
A third limitation is associated with the information on the babies' ethnicity registered in the NN4B data. Ethnicity of the baby is reported by the mother and while the classification of white or nonwhite births seems to be consistent between self-reported and health databases, specific minority groups may be misclassified depending on how the mother defines ethnicity (39).

Finally, given that birth weight trends are known to change over time(26), we recommend that such analyses are updated periodically.

\section{Conclusions}

In conclusion, we compiled birth weight centile charts based on over 4 million live singleton births for the main ethnic groups in England and Wales, including the $10^{\text {th }}$ centiles for defining small for gestational age. Using sex only centiles that do not also take account of ethnicity can lead to SGA misclassification. National reference birth weight charts should account for ethnic group to better represent the diverse population of England and Wales. The centiles can be used by researchers to determine SGA by ethnic group to avoid the misclassification of babies born small or large for gestational age, which may be particularly useful in epidemiological studies. 


\section{References}

1. Wright CM, Williams AF, Elliman D, Bedford H, Birks E, Butler G, et al. Using the new UK-WHO growth charts. BMJ. 2010;340.

2. Wilcox M, Gardosi J, Mongelli M, Ray C, Johnson I. Birth weight from pregnancies dated by ultrasonography in a multicultural British population. BMJ. 1993;307:588-91.

3. Wright CM, Booth IW, Buckler JMH, Cameron N, Cole TJ, Healy MJR, et al. Growth reference charts for use in the United Kingdom. Archives of Disease in Childhood. 2002;86(1):11-4.

4. Cole TJ, Freeman JV, Preece MA. British 1990 growth reference centiles for weight, height, body mass index and head circumference fitted by maximum penalized likelihood. Statistics in Medicine. 1998;17(4):407-29.

5. Cole TJ, Williams AF, Wright CM. Revised birth centiles for weight, length and head circumference in the UK-WHO growth charts. Ann Hum Biol. 2011;38(1):7-11.

6. Kierans WJ, Joseph KS, Luo ZC, Platt R, Wilkins R, Kramer MS. Does one size fit all? The case for ethnic-specific standards of fetal growth. BMC Pregnancy Childbirth. 2008;8:1.

7. Hanley GE, Janssen PA. Ethnicity-specific birthweight distributions improve identification of term newborns at risk for short-term morbidity. Am J Obstet Gynecol. 2013;209(5):428.e1-6.

8. Kelly Y, Panico L, Bartley M, Marmot M, Nazroo J, Sacker A. Why does birthweight vary among ethnic groups in the UK? Findings from the Millennium Cohort Study. Journal of Public Health. 2008;31(1):131-7.

9. de Wilde JA, van Buuren S, Middelkoop BJC. Trends in birth weight and the prevalence of low birth weight and small-for-gestational-age in Surinamese South Asian babies since 1974: cross-sectional study of three birth cohorts. BMC Public Health. 2013;13(1):931.

10. Ray JG, Jiang D, Sgro M, Shah R, Singh G, Mamdani MM. Thresholds for Small for Gestational Age Among Newborns of East Asian and South Asian Ancestry. Journal of Obstetrics and Gynaecology Canada. 2009;31(4):322-30.

11. Norris T, Johnson W, Farrar D, Tuffnell D, Wright J, Cameron N. Small-for-gestational age and large-for-gestational age thresholds to predict infants at risk of adverse delivery and neonatal outcomes: are current charts adequate? An observational study from the Born in Bradford cohort. BMJ Open. 2015;5(3).

12. West J, Lawlor DA, Fairley L, Wright J. P17 Birth size differences between white and Pakistani origin infants by generation: results from the born in Bradford cohort study. Journal of Epidemiology and Community Health. 2010;64(Suppl 1):A40.

13. Fairley L, Petherick ES, Howe LD, Tilling K, Cameron N, Lawlor DA, et al. Describing differences in weight and length growth trajectories between white and Pakistani infants in the UK: analysis of the Born in Bradford birth cohort study using multilevel linear spline models. Archives of Disease in Childhood. 2013;98(4):274.

14. Larkin JC, Naimi Al. Effect of Population-Specific Birthweight Curves on Disparities in Perinatal Mortality in Small-for-Gestational Age Pregnancies. Am J Perinatol. 2018;35(7):695-702.

15. Figueras F, Figueras J, Meler E, Eixarch E, Coll O, Gratacos E, et al. Customised birthweight standards accurately predict perinatal morbidity. Archives of Disease in Childhood - Fetal and Neonatal Edition. 2007;92(4):F277.

16. Gardosi J. Customized Charts and Their Role in Identifying Pregnancies at Risk Because of Fetal Growth Restriction. Journal of Obstetrics and Gynaecology Canada. 2014;36(5):408-15.

17. Jiang D, Singh G, Ray J, Sgro M, Mamdani MM, Shah R. Birthweight Curves and Thresholds for Small for Gestational Age Tailored to Newborns of East Asian and South Asian Ancestry. Paediatrics \& Child Health. 2009;14(suppl_A):37A-A. 
18. Ray JG, Sgro M, Mamdani MM, Glazier RH, Bocking A, Hilliard R, et al. Birth Weight Curves Tailored to Maternal World Region. Journal of Obstetrics and Gynaecology Canada. 2012;34(2):159-71.

19. Seaton SE, Yadav KD, Field DJ, Khunti K, Manktelow BN. Birthweight centile charts for South Asian infants born in the UK. Neonatology. 2011;100(4):398-403.

20. Candela S, Ranzi A, Bonvicini L, Baldacchini F, Marzaroli P, Evangelista A, et al. Air Pollution from Incinerators and Reproductive Outcomes: A Multisite Study. Epidemiology. 2013;24(6):863-70.

21. Ghosh RE, Freni-Sterrantino A, Douglas P, Parkes B, Fecht D, de Hoogh K, et al. Fetal growth, stillbirth, infant mortality and other birth outcomes near UK municipal waste incinerators; retrospective population based cohort and case-control study. Environment International. 2019;122:151-8.

22. Hilder L, Moser K, Dattani N, Macfarlane A. Pilot linkage of NHS Numbers for Babies data with birth registrations. Health Stat Q. 2007(33):25-33.

23. Tukey J. Exploratory Data Analysis. The Concise Encyclopedia of Statistics. New York, NY: Springer New York; 2008. p. 192-4.

24. Rigby RA, Stasinopoulos DM. The GAMLSS project: a Flexible Approach to Statistical Modelling. In: Klein B, Korsholm L, editors. New Trends in Statistical Modelling: Proceeding of the 16th International Worksop on Statistical Modelling; Odense, Denmark2001. p. 337-45.

25. Norris T, Seaton SE, Manktelow BN, Baker PN, Kurinczuk JJ, Field D, et al. Updated birth weight centiles for England and Wales. Archives of Disease in Childhood - Fetal and Neonatal Edition. 2017.

26. Ghosh RE, Berild JD, Sterrantino AF, Toledano MB, Hansell AL. Birth weight trends in England and Wales (1986-2012): babies are getting heavier. Arch Dis Child Fetal Neonatal Ed. 2017.

27. Maso G, Jayawardane MAMM, Alberico S, Piccoli M, Senanayake HM. The Implications of Diagnosis of Small for Gestational Age Fetuses Using European and South Asian Growth Charts: An Outcome-Based Comparative Study. The Scientific World Journal. 2014;2014:5.

28. Narchi H, Skinner A, Williams B. Small for gestational age neonates - are we missing some by only using standard population growth standards and does it matter? The Journal of Maternal-Fetal \& Neonatal Medicine. 2010;23(1):48-54.

29. Kramer MS, Ananth CV, Platt RW, Joseph K. US Black vs White disparities in foetal growth: physiological or pathological? International Journal of Epidemiology. 2006;35(5):1187-95.

30. Alexander GR, Kogan MD, Himes JH, Mor JM, Goldenberg R. Racial differences in birthweight for gestational age and infant mortality in extremely-low-risk US populations. Paediatric and perinatal epidemiology. 1999;13(2):205-17.

31. Joseph KS, Wilkins R, Dodds L, Allen VM, Ohlsson A, Marcoux S, et al. Customized birth weight for gestational age standards: Perinatal mortality patterns are consistent with separate standards for males and females but not for blacks and whites. BMC Pregnancy and Childbirth. 2005;5(1):3.

32. Margetts BM, Mohd Yusof S, Al Dallal Z, Jackson AA. Persistence of lower birth weight in second generation South Asian babies born in the United Kingdom. Journal of Epidemiology and Community Health. 2002;56(9):684.

33. Leon DA, Moser KA. Low birth weight persists in South Asian babies born in England and Wales regardless of maternal country of birth. Slow pace of acculturation, physiological constraint or both? Analysis of routine data. Journal of Epidemiology and Community Health. 2012;66(6):544.

34. Cruickshank JK, Rosato MG, Harding S. Lack of change in birthweights of infants by generational status among Indian, Pakistani, Bangladeshi, Black Caribbean, and Black African mothers in a British cohort study. International Journal of Epidemiology. 2004;33(6):1279-85.

35. Catov JM, Lee M, Roberts JM, Xu J, Simhan HN. Race Disparities and Decreasing Birth Weight: Are All Babies Getting Smaller? American Journal of Epidemiology. 2016;183(1):15-23.

36. Kramer MS, Platt RW, Wen SW, Joseph KS, Allen A, Abrahamowicz M, et al. A new and improved population-based Canadian reference for birth weight for gestational age. Pediatrics. 2001;108(2):E35. 
37. West J, Lawlor DA, Fairley L, Bhopal R, Cameron N, McKinney PA, et al. UK-born Pakistani-origin infants are relatively more adipose than white British infants: findings from 8704 mother-offspring pairs in the Born-in-Bradford prospective birth cohort. Journal of Epidemiology and Community Health. 2013;67(7):544.

38. David RJ, Collins JW. Differing Birth Weight among Infants of U.S.-Born Blacks, African-Born Blacks, and U.S.-Born Whites. The New England Journal of Medicine. 1997;337(17):1209-14.

39. Saunders CL, Abel GA, El Turabi A, Ahmed F, Lyratzopoulos G. Accuracy of routinely recorded ethnic group information compared with self-reported ethnicity: evidence from the English Cancer Patient Experience survey. BMJ Open. 2013;3(6). 



\section{Tables and Figures:}

Table 1: Distribution by ethnicity and sex of live singleton births between 24 and 42 weeks' gestational age, by number and percentage after removing outliers. Number of observations ( $\mathrm{n}$ ) after outlier's removal, and percentage (\%), mean and standard deviation of birth weight by sex and ethnicity for eligible births in UK-EBWC from 2006-2012.

\begin{tabular}{|c|c|c|c|c|c|c|c|c|c|c|}
\hline Ethnicity & \multicolumn{2}{|c|}{ White } & \multicolumn{2}{|c|}{ Black } & \multicolumn{2}{|c|}{ Asian } & \multicolumn{2}{|c|}{ South Asian ${ }^{1}$} & \multicolumn{2}{|c|}{ Other Asian ${ }^{1}$} \\
\hline Sex & Male & Female & Male & Female & Male & Female & Male & Female & Male & Female \\
\hline $\mathrm{n}$ & $1,735,148$ & $1,643,808$ & 119,741 & 116,781 & 229,423 & 219,680 & 193,660 & 185,752 & 35,770 & 33,961 \\
\hline$\%$ & 74.99 & 74.42 & 5.18 & 5.31 & 9.92 & 9.99 & 8.37 & 8.44 & 1.55 & 1.54 \\
\hline Mean birth weight (g) & 3470 & 3345 & 3342 & 3221 & 3186 & 3094 & 3171 & 3079 & 3269 & 3181 \\
\hline Standard deviation (g) & 562 & 533 & 580 & 566 & 533 & 509 & 532 & 508 & 532 & 508 \\
\hline Sex difference (g) & \multicolumn{2}{|c|}{125} & \multicolumn{2}{|c|}{121} & \multicolumn{2}{|c|}{92} & \multicolumn{2}{|c|}{92} & \multicolumn{2}{|c|}{88} \\
\hline
\end{tabular}

1 South Asian (Indian, Pakistani and Bangladeshi) and other Asian (any other Asian background) are two subsets derived from Asian. 
Table 2: Observed and expected percentages below selected centiles by sex.

\begin{tabular}{lccccc}
\hline & Expected & Males & $\begin{array}{c}\text { Risk } \\
\text { Difference }\end{array}$ & Females & $\begin{array}{c}\text { Risk } \\
\text { Difference }\end{array}$ \\
\hline $0.4^{\text {th }}$ & 0.38 & 0.44 & 0.06 & 0.46 & 0.08 \\
$2^{\text {nd }}$ & 2.28 & 2.03 & -0.25 & 2.05 & -0.23 \\
$9^{\text {th }}$ & 9.13 & 8.76 & -0.37 & 8.76 & -0.37 \\
$10^{\text {th }}$ & 10 & 9.81 & -0.19 & 9.70 & -0.30 \\
$25^{\text {th }}$ & 25.24 & 24.76 & -0.48 & 24.72 & -0.52 \\
$50^{\text {th }}$ & 50 & 50.29 & 0.29 & 49.81 & -0.19 \\
$75^{\text {th }}$ & 74.76 & 75.34 & 0.58 & 74.91 & 0.15 \\
$91^{\text {st }}$ & 90.67 & 90.96 & 0.29 & 90.81 & 0.14 \\
$98^{\text {th }}$ & 97.72 & 97.83 & 0.11 & 97.78 & 0.06 \\
$99.6^{\text {th }}$ & 99.62 & 99.60 & -0.02 & 99.61 & -0.01 \\
\hline
\end{tabular}


Figure 1: UK-EBWC 2006-12 birth weight centiles distribution for White, Black and Asian births compared with UK-EBWC, by sex.

Figure 2: UK-EBWC birth weight centiles for White, Black and Asian births compared with UKEBWC sex-specific.

Figure 3 UK-EBWC birth weight $10^{\text {th }}$ centiles for ethnic-specific males compared with the sexspecific centile. 